\title{
ON REGULAR VARIATION FOR \\ INFINITELY DIVISIBLE RANDOM VECTORS AND ADDITIVE PROCESSES
}

\author{
HENRIK HULT, ${ }^{*}$ Cornell University \\ FILIP LINDSKOG, ${ }^{* *}$ KTH Stockholm
}

\begin{abstract}
We study the tail behavior of regularly varying infinitely divisible random vectors and additive processes, i.e. stochastic processes with independent but not necessarily stationary increments. We show that the distribution of an infinitely divisible random vector is tail equivalent to its Lévy measure and we study the asymptotic decay of the probability for an additive process to hit sets far away from the origin. The results are extensions of known univariate results to the multivariate setting; we exemplify some of the difficulties that arise in the multivariate case.
\end{abstract}

Keywords: Multivariate regular variation; infinitely divisible distribution; additive process; Lévy process

2000 Mathematics Subject Classification: Primary 60G51

Secondary 60G70; 60E07

\section{Introduction}

Stochastic processes with independent increments and heavy-tailed marginal distributions have become increasingly popular for studying problems in areas such as insurance mathematics, mathematical finance, and communications networks. Efforts have been made to understand the extremal behavior of such processes and to find the tail behavior of functionals of their sample paths; see, e.g. [5], [7], and [20]. However, whereas problems concerning the tail behavior of univariate stochastic processes have been studied successfully for quite some time, multivariate processes have received far less attention. One possible reason is that, except for regular variation, the univariate notions of heavy tails do not have natural multivariate versions. In particular there is no well-established theory of multivariate subexponentiality, although we acknowledge the developments in [6]. Heavy-tailed random vectors are therefore often studied either under an assumption of multivariate regular variation or for a particular choice of parametric model.

In this paper we study tails of infinitely divisible random vectors and extend well-known univariate results on the tail behavior to the multivariate setting, under an assumption of multivariate regular variation. We illustrate, by example, several difficulties that appear in the multivariate setting due to the dependence structure of the random vector. Closely related are additive processes (see [21, p. 3]), since $\boldsymbol{X}_{t}$ is infinitely divisible for every $t \in[0, T]$ if $\left(\boldsymbol{X}_{t}\right)_{t \in[0, T]}$ is an additive process. Under the condition that $\boldsymbol{X}_{T}$ be regularly varying, we study the asymptotics of the probability of hitting sets far away from the origin during a fixed time

Received 5 February 2003; revision received 19 July 2005.

* Postal address: School of Operations Research and Industrial Engineering, Cornell University, 414A Rhodes Hall, Ithaca, NY 14853, USA. Email address: hh228@cornell.edu

** Postal address: Department of Mathematics, KTH, 10044 Stockholm, Sweden. 
interval $[0, T]$. The intuitive idea is that a heavy-tailed process with independent increments reaches a set far away from the origin by making one big jump to that set. The asymptotic decay (as $u \rightarrow \infty$ ) of the probability for an additive process to hit a set $u A$ during an arbitrary subset $S$ of $[0, T]$ is also considered in this paper. It is worth mentioning that the heuristic idea of making 'one big jump' to reach sets far away can be rigorously formulated within the framework of regular variation on the space of functions that are continuous from the right with left limits (càdlàg) (see [9]). This notion has also proven useful in studying the tail behavior of functionals of sample paths of heavy-tailed multivariate stochastic processes. For more details we refer the reader to [8], [9], [12], and [10]. Because of these recent developments, we do not enter into a deeper study of the tail behavior of functionals of additive processes. In this paper we choose not to work within the framework of regular variation on the space of càdlàg functions, since it is not necessary for the problems we consider here. We also feel that this choice makes the paper more accessible.

In Section 2 we recall the notion of multivariate regular variation. In Section 3 we give a multivariate version of the result in [7] which says that the distribution of an infinitely divisible random variable and its Lévy measure are tail equivalent. We discuss difficulties that necessarily arise when going from the univariate to the multivariate setting. In Section 4 we study the tail behavior of multivariate additive processes and give a multivariate version of the result in [22] which says that the distribution of the supremum of a univariate Lévy process over $[0, T]$ is tail equivalent to the distribution of the process at time $T$. Section 5 contains the proofs of our results. In Appendix A we give useful auxiliary results that are needed in these proofs.

\section{Preliminaries}

We assume that all random elements considered are defined on a common probability space $(\Omega, \mathcal{F}, \mathrm{P})$. The $\sigma$-algebra of Borel subsets of a topological space $\mathbb{E}$ is denoted by $\mathcal{B}(\mathbb{E})$. For a set $A \in \mathscr{B}(\mathbb{E})$, we denote by $A^{\circ}$ and $\bar{A}$ the interior and the closure of $A$, respectively. Moreover, $\partial A=\bar{A} \backslash A^{\circ}$ is the boundary of $A$.

Regular variation of random vectors is usually formulated in terms of vague convergence of Radon measures on $\overline{\mathbb{R}}^{d} \backslash\{\boldsymbol{0}\}$, where $\overline{\mathbb{R}}=[-\infty, \infty]$. The space $\overline{\mathbb{R}}^{d} \backslash\{\boldsymbol{0}\}$ is equipped with the usual topology (see, e.g. [17]), so that the Borel sets we are interested in are the usual ones, i.e.

$$
\mathscr{B}\left(\overline{\mathbb{R}}^{d} \backslash\{\mathbf{0}\}\right) \cap\left(\mathbb{R}^{d} \backslash\{\mathbf{0}\}\right)=\mathscr{B}\left(\mathbb{R}^{d}\right) \cap\left(\mathbb{R}^{d} \backslash\{\mathbf{0}\}\right),
$$

and the Borel sets of $\mathbb{R}^{d}$ bounded away from $\mathbf{0}$ are relatively compact in $\overline{\mathbb{R}}^{d} \backslash\{\mathbf{0}\}$.

An $\mathbb{R}^{d}$-valued random vector $\boldsymbol{X}=\left(X^{(1)}, \ldots, X^{(d)}\right)$ with unbounded support is regularly varying if there exist an $\alpha>0$, a function $L$ that is slowly varying at infinity, and a nonzero Radon measure $\mu$ defined on $\mathcal{B}\left(\overline{\mathbb{R}}^{d} \backslash\{\mathbf{0}\}\right)$ with $\mu\left(\overline{\mathbb{R}}^{d} \backslash \mathbb{R}^{d}\right)=0$ such that, as $u \rightarrow \infty$,

$$
u^{\alpha} L(u) \mathrm{P}\left(u^{-1} \boldsymbol{X} \in \cdot\right) \stackrel{\mathrm{v}}{\rightarrow} \mu(\cdot) \quad \text { on } \mathcal{B}\left(\overline{\mathbb{R}}^{d} \backslash\{\mathbf{0}\}\right) .
$$

Here ' $\stackrel{\text { v }}{\rightarrow}$ ' denotes vague convergence; see, e.g. [13, p. 169] or [19, p. 139]. If (2.1) holds then $\mu(x B)=x^{-\alpha} \mu(B)$ for every $x>0$ and $B \in \mathscr{B}\left(\overline{\mathbb{R}}^{d} \backslash\{\mathbf{0}\}\right)$ (see, e.g. [14, Theorem 1.14]), and we write $\boldsymbol{X} \in \operatorname{RV}(\alpha, L, \mu)$. For a Radon measure $v$ on $\mathscr{B}\left(\overline{\mathbb{R}}^{d} \backslash\{\boldsymbol{0}\}\right)$ we similarly write $v \in \mathrm{RV}(\alpha, L, \mu)$ if $u^{\alpha} L(u) v(u \cdot) \stackrel{\mathrm{v}}{\rightarrow} \mu(\cdot)$ on $\mathscr{B}\left(\overline{\mathbb{R}}^{d} \backslash\{\mathbf{0}\}\right)$.

Remark 2.1. (i) The homogeneity property of the limit measure $\mu$ implies that $\mu$ assigns no mass to spheres centered at the origin, i.e. sets of the form $\left\{\boldsymbol{y} \in \mathbb{R}^{d}:|\boldsymbol{y}|=r\right\}$ for $r>0$, with respect to any norm $|\cdot|$ on $\mathbb{R}^{d}$. In particular, $\mu$ has no atoms (see [1]). 
(ii) Condition (2.1) is equivalent to convergence for every set $A \in \mathcal{B}\left(\mathbb{R}^{d}\right)$ bounded away from 0 (i.e. $0 \notin \bar{A}$ ) with $\mu(\partial A)=0$ (see [13, Theorem 15.7.2, p. 169]).

(iii) Several equivalent formulations of multivariate regular variation can be found in, e.g. [1], [2], [14], and [17].

\section{Tails of regularly varying infinitely divisible random vectors}

Let $X$ be a nonnegative infinitely divisible random variable with Lévy measure $v$. It was shown in [7] that the following statements are equivalent:

(i) $\mathrm{P}(X \leq u)$ is subexponential;

(ii) $v(1, u] / v(1, \infty)$ is subexponential;

(iii) $\mathrm{P}(X>u) / v(u, \infty) \rightarrow 1$ as $u \rightarrow \infty$.

In particular, $X \in \operatorname{RV}(\alpha, L, \mu)$ if and only if $v \in \operatorname{RV}(\alpha, L, \mu)$. Satisfaction of the condition in statement (iii) is often referred to as the distribution of $X$ being tail equivalent to its Lévy measure. This equivalence is fundamental when studying the extremal behavior of heavy-tailed processes with independent increments and functionals of the sample paths of such processes (see, e.g. [20]). The following result extends this tail equivalence to regularly varying infinitely divisible random vectors.

Let $\boldsymbol{X}$ denote an infinitely divisible $\mathbb{R}^{d}$-valued random vector with Lévy measure $v$.

Proposition 3.1. $X \in \operatorname{RV}(\alpha, L, \mu)$ if and only if $v \in \operatorname{RV}(\alpha, L, \mu)$.

It is worth mentioning that if the index of regular variation, $\alpha$, is not an integer, then Proposition 3.1 can easily be proved by combining results of [2] and [7]. This can be seen from the following argument. If $\boldsymbol{X} \in \operatorname{RV}(\alpha, L, \mu)$ then, for every $u>0$ and $\boldsymbol{x} \neq \mathbf{0}$,

$$
\lim _{u \rightarrow \infty} u^{\alpha} L(u) \mathrm{P}(\langle\boldsymbol{x}, \boldsymbol{X}\rangle>u)=\lim _{u \rightarrow \infty} u^{\alpha} L(u) \mathrm{P}\left(\boldsymbol{X} \in u W_{\boldsymbol{x}}\right)=\mu\left(W_{\boldsymbol{x}}\right),
$$

where $\langle\boldsymbol{x}, \boldsymbol{X}\rangle=x^{(1)} X^{(1)}+\cdots+x^{(d)} X^{(d)}$ and $W_{\boldsymbol{x}}=\left\{\boldsymbol{y} \in \mathbb{R}^{d}:\langle\boldsymbol{x}, \boldsymbol{y}\rangle>1\right\}$. Arguing from Theorem 1 of [7] if $\mu\left(W_{\boldsymbol{x}}\right)>0$ (i.e. the random variable $\langle\boldsymbol{x}, \boldsymbol{X}\rangle$ is regularly varying) and some additional arguments if $\mu\left(W_{\boldsymbol{x}}\right)=0$ (i.e. the random variable $\langle\boldsymbol{x}, \boldsymbol{X}\rangle$ has lighter tails), it follows that, for every $\boldsymbol{x} \neq \mathbf{0}$,

$$
\lim _{u \rightarrow \infty} u^{\alpha} L(u) v\left(u W_{x}\right)=\mu\left(W_{x}\right) .
$$

If $\alpha$ is not an integer then Theorem 1.1 of [2] implies that $v \in \operatorname{RV}(\alpha, L, \mu)$. The converse can be shown similarly.

However, if the index of regular variation is an integer, then Proposition 3.1 cannot be proved in this way. The reason is that if $\alpha$ is an integer, then regular variation of all linear combinations does not necessarily imply multivariate regular variation. This was recently shown in [11]. It may be noted that the random vector in the example of [11] is not infinitely divisible. However, an example in which the random vector is infinitely divisible is easily constructed. If we let $v$ be the distribution of the random vector considered in Section 2.2 of [11], then an infinitely divisible random vector $\boldsymbol{X}$ with Lévy measure $v$ has the following properties:

(i) $\boldsymbol{X}$ is not regularly varying,

(ii) $\langle\boldsymbol{x}, \boldsymbol{X}\rangle$ is regularly varying for every $\boldsymbol{x} \neq \mathbf{0}$.

In Section 5 we give a direct proof of Proposition 3.1 that is valid for all $\alpha>0$. 
The following corollary is a consequence of Proposition 3.1.

Corollary 3.1. Let $\boldsymbol{X}$ be an infinitely divisible $\mathbb{R}^{d}$-valued random vector with Lévy measure $v$, satisfying $\boldsymbol{X} \in \mathrm{RV}(\alpha, L, \mu)$. If $A \in \mathcal{B}\left(\mathbb{R}^{d}\right)$ is bounded away from $\mathbf{0}$ with $\mu(A)>0$ and $\mu(\partial A)=0$, then $\lim _{u \rightarrow \infty} v(u A) / \mathrm{P}(\boldsymbol{X} \in u A)=1$.

The following example shows that the tail equivalence does not hold in general without the condition $\mu(A)>0$.

Example 3.1. Let $\boldsymbol{X}$ be an $\mathbb{R}^{2}$-valued infinitely divisible random vector with independent, purely non-Gaussian symmetric components. Suppose that $\boldsymbol{X} \in \operatorname{RV}(\alpha, L, \mu)$ with $\alpha>1$. Then the characteristic function of $\boldsymbol{X}$ is (see [21, p. 39])

$$
\mathrm{E}(\exp \{\mathrm{i}\langle\boldsymbol{x}, \boldsymbol{X}\rangle\})=\int_{\mathbb{R}^{2}}(\exp \{\mathrm{i}\langle\boldsymbol{x}, \boldsymbol{z}\rangle\}-1) v(\mathrm{~d} \boldsymbol{z}) .
$$

Since

$$
\begin{aligned}
\mathrm{E}(\exp \{\mathrm{i}\langle\boldsymbol{x}, \boldsymbol{X}\rangle\}) & =\mathrm{E}\left(\exp \left\{\mathrm{i} x^{(1)} X^{(1)}\right\}\right) \mathrm{E}\left(\exp \left\{\mathrm{i} x^{(2)} X^{(2)}\right\}\right) \\
& =\left(\int_{\mathbb{R}}\left(\exp \left\{\mathrm{i} x^{(1)} z\right\}-1\right) \nu_{1}(\mathrm{~d} z)\right)\left(\int_{\mathbb{R}}\left(\exp \left\{\mathrm{i} x^{(2)} z\right\}-1\right) \nu_{2}(\mathrm{~d} z)\right)
\end{aligned}
$$

where $v_{k}$ is the Lévy measure of $X^{(k)}, k=1,2$, it follows that the Lévy measure $v$ of $\boldsymbol{X}$ concentrates on the coordinate axes. In particular, for every $u>0, v((u, \infty) \times(u, \infty))=0$ and

$$
\mathrm{P}(X \in(u, \infty) \times(u, \infty))=\mathrm{P}\left(X^{(1)}>u\right) \mathrm{P}\left(X^{(2)}>u\right)>0 .
$$

\section{Hitting probabilities for additive processes}

We consider an $\mathbb{R}^{d}$-valued additive process $\boldsymbol{X}=\left(\boldsymbol{X}_{t}\right)_{t \in[0, T]}$ (see [21, p. 3]), where $T>0$ is fixed. We always assume that $\boldsymbol{X}$ denotes the unique càdlàg version of the process. Recall that $\boldsymbol{X}_{t}$ is infinitely divisible for each $t \in[0, T]$ and that the process is completely determined by a family $\left\{\left(A_{t}, v_{t}, \gamma_{t}\right)\right\}$ of generating triplets. As in [21, p. 53], we denote by $\tilde{v}$ the measure on $\mathcal{B}\left([0, T] \times \mathbb{R}^{d} \backslash\{\mathbf{0}\}\right)$ given by $\tilde{v}([0, t] \times \cdot)=v_{t}(\cdot)$.

We assume that $\boldsymbol{X}_{T} \in \mathrm{RV}\left(\alpha, L, \mu_{T}\right)$. By Proposition 3.1, this is equivalent to having $v_{T} \in \mathrm{RV}\left(\alpha, L, \mu_{T}\right)$. First consider a Lévy process $\boldsymbol{X}$, i.e. an additive process with stationary increments. The Lévy measure $v_{t}$ of $\boldsymbol{X}_{t}$ is then given by $v_{t}=t v_{1}$, and the following statement holds:

$$
\boldsymbol{X}_{1} \in \operatorname{RV}\left(\alpha, L, \mu_{1}\right) \quad \text { if and only if } \quad \boldsymbol{X}_{t} \in \operatorname{RV}\left(\alpha, L, t \mu_{1}\right) \quad \text { for every } t>0 .
$$

Thus, to verify that $\boldsymbol{X}_{t}$ is regularly varying for arbitrary $t>0$, it is enough to check that $\boldsymbol{X}_{1}$ is regularly varying. For additive processes things are less straightforward. As we will see in the next example, we can construct an additive process $X=\left(X_{t}\right)_{t \in[0, T]}$ such that $X_{T} \in \mathrm{RV}\left(\alpha, L, \mu_{T}\right)$ but $X_{t}$ is not regularly varying for $t<T$; in fact, for every $t<T$, the process $\left(X_{S}\right)_{S \in[0, t]}$ has bounded jumps.

Example 4.1. Let $\tilde{v}$ be a probability measure on $[0, T] \times[1, \infty)$ given by

$$
\tilde{v}(\mathrm{~d}(t, x))=\sum_{k=1}^{\infty} \frac{k}{T} 1_{(T-T / k, T]}(t) 1_{[k, k+1)}(x) \alpha x^{-\alpha-1} \mathrm{~d}(t, x) .
$$


Let $\xi$ be a Poisson random measure with intensity measure $\tilde{v}$ and let $X=\left(X_{t}\right)_{t \in[0, T]}$ be a stochastic process given by

$$
X_{t}=\int_{[0, t] \times[1, \infty)} x \xi(\mathrm{d}(s, x)), \quad t \in[0, T] .
$$

The following argument shows that $X$ is an additive process. For any $s<t$ with $s, t \in[0, T]$ and $t-s<1 / m$, we have

$$
\begin{aligned}
\tilde{v}([s, t] \times[1, \infty)) & \leq \tilde{v}([T-1 / m, T] \times[1, \infty)) \\
& =\sum_{k=1}^{\infty} \int_{T-1 / m}^{T} \frac{k}{T} 1_{(T-T / k, T]}(t) \mathrm{d} t \int_{1}^{\infty} 1_{[k, k+1)}(x) \alpha x^{-\alpha-1} \mathrm{~d} x \\
& =\sum_{k=1}^{\infty} \min \left(\frac{k}{T m}, 1\right)\left(k^{-\alpha}-(k+1)^{-\alpha}\right) \\
& \leq\lfloor T m\rfloor^{-\alpha}+\sum_{k=1}^{\lfloor T m\rfloor} \frac{k}{T m}\left(k^{-\alpha}-(k+1)^{-\alpha}\right) \\
& \rightarrow 0 \text { as } m \rightarrow \infty,
\end{aligned}
$$

where $\lfloor m\rfloor$ is the integer part of $m$. Hence, for $s<t, \tilde{v}([s, t] \times[1, \infty)) \rightarrow 0$ as $|t-s| \rightarrow 0$. Thus, for any $\varepsilon>0$,

$$
\mathrm{P}\left(\left|X_{t}-X_{s}\right|>\varepsilon\right) \leq \mathrm{P}(\xi([s, t] \times[1, \infty))>0)=1-\exp \{-\tilde{v}([s, t] \times[1, \infty))\} \rightarrow 0
$$

as $|t-s| \rightarrow 0$, i.e. $X$ is stochastically continuous. In addition, by Proposition 19.5 of [21, p. 123], for disjoint sets $S_{1}, \ldots, S_{k} \in \mathscr{B}([0, T])$,

$$
\int_{S_{1} \times[1, \infty)} x \xi(\mathrm{d}(s, x)), \quad \ldots, \quad \int_{S_{k} \times[1, \infty)} x \xi(\mathrm{d}(s, x))
$$

are independent, i.e. $X$ has independent increments. Moreover, by construction $X(\omega)$ is right continuous with left limits for every $\omega \in \Omega$. Finally, $X_{0}=0$ almost surely. Hence, $X$ is an additive process. By Proposition 19.5 of [21], for every $t \in[0, T], X_{t}$ has Lévy measure $v_{t}(\cdot)=\tilde{v}([0, t] \times \cdot)$. Choose $x, u>0$ with $x u>1$ and note that

$$
v_{T}(x u, \infty)=\int_{x u}^{\infty} \alpha y^{-\alpha-1} \mathrm{~d} y=(x u)^{-\alpha}
$$

and, for $t<T$ and sufficiently large $u, v_{t}(x u, \infty)=0$.

We now study the asymptotics of the probability of hitting a set $u A$ far away from $\mathbf{0}$ at some $t \in[0, T], T>0$, i.e. we study $\mathrm{P}\left(\boldsymbol{X}_{t} \in u A\right.$ for some $\left.t \in[0, T]\right)$ as $u \rightarrow \infty$. Notice that if the process is univariate and $A=(1, \infty)$, then this corresponds to studying the decay of $\mathrm{P}\left(\sup _{t \in[0, T]} X_{t}>u\right)$ as $u \rightarrow \infty$. The intuition behind the result is that the process reaches the set $u A$ in one big jump. In comparison to the big jump, the process does not move much before or after the jump. Therefore, we expect that the probability of hitting a set $u A$ during $[0, T]$ decays as the probability of being in the set at time $T$. This is indeed the case. 
Proposition 4.1. Let $\left(\boldsymbol{X}_{t}\right)_{t \in[0, T]}$ be an $\mathbb{R}^{d}$-valued additive process satisfying

$$
\boldsymbol{X}_{T} \in \mathrm{RV}\left(\alpha, L, \mu_{T}\right) .
$$

If $A \in \mathscr{B}\left(\mathbb{R}^{d}\right)$ is bounded away from $\mathbf{0}$, with $\mu_{T}(A)>0$ and $\mu_{T}(\partial A)=0$, then

$$
\lim _{u \rightarrow \infty} \frac{\mathrm{P}\left(\boldsymbol{X}_{t} \in u A \text { for some } t \in[0, T]\right)}{\mathrm{P}\left(\boldsymbol{X}_{T} \in u A\right)}=1 .
$$

Remark 4.1. (i) Notice that it is sufficient to assume that $\boldsymbol{X}_{T}$ is regularly varying; we do not need assumptions on the distribution of $\boldsymbol{X}_{t}$ for $t<T$.

(ii) It was shown in [22] that if $\left(X_{t}\right)_{t \in[0, T]}$ is a univariate Lévy process and if $X_{T}$ is long tailed, i.e. $\lim _{u \rightarrow \infty} \mathrm{P}\left(X_{T}>u-y\right) / \mathrm{P}\left(X_{T}>u\right)=1$ for every $y \in \mathbb{R}$, then

$$
\mathrm{P}\left(\sup _{t \in[0, T]} X_{t}>u\right) / \mathrm{P}\left(X_{T}>u\right) \rightarrow 1
$$

as $u \rightarrow \infty$. Hence, Proposition 4.1 holds for sets $A=\left\{x \in \mathbb{R}^{d}: x^{(k)}>c\right\}$, with $c>0$ and $k \in\{1, \ldots, d\}\}$, under the weaker condition that the components of $\boldsymbol{X}_{T}$ are long tailed. However, it is not obvious for which more general sets this remains true.

There are many situations in which we need more information about the process $\boldsymbol{X}$ than just the tail behavior of $\boldsymbol{X}_{T}$. For instance, if we are interested in the tail behavior of certain functionals of the sample paths of the process, then we typically need to know something about the tail behavior of $\boldsymbol{X}_{t}$ for $t<T$. The same is true if we are interested in the asymptotics of the distribution of the time of the big jump that determines the extremal behavior of the process.

To address these questions we take the following approach. We assume that $\boldsymbol{X}_{T} \in$ $\mathrm{RV}\left(\alpha, L, \mu_{T}\right)$ and that, for each $t$ in a set $U_{T} \subset[0, T]$ whose complement is at most countable, there exists a Radon measure $\mu_{t}$ on $\mathcal{B}\left(\overline{\mathbb{R}}^{d} \backslash\{\mathbf{0}\}\right)$, with $\mu_{t}\left(\overline{\mathbb{R}}^{d} \backslash \mathbb{R}^{d}\right)=0$, such that $u^{\alpha} L(u) \mathrm{P}\left(u^{-1} \boldsymbol{X}_{t} \in \cdot\right) \stackrel{\mathrm{V}}{\rightarrow} \mu_{t}(\cdot)$. Here $\mu_{t}$ may be the null measure for $t<T$. The assumption implies that, for $t<T$, the random vector $\boldsymbol{X}_{t}$ is either regularly varying (if $\mu_{t}$ is nonzero) with the same index $\alpha$ and slowly varying function $L$ as $\boldsymbol{X}_{T}$, or that the vague limit of $\left(u^{\alpha} L(u) \mathrm{P}\left(u^{-1} \boldsymbol{X}_{t} \in \cdot\right)\right)_{u>0}$ as $u \rightarrow \infty$ is the null measure, i.e. that $\boldsymbol{X}_{t}$ has lighter tails than $\boldsymbol{X}_{T}$. Under these assumptions we can define a measure $\tilde{\mu}$ on $\mathcal{B}\left([0, T] \times \overline{\mathbb{R}}^{d} \backslash\{\boldsymbol{0}\}\right)$ that is determined by $\tilde{\mu}([0, t] \times A)=\mu_{t}(A)$ for every $t \in U_{T}$ and $A \in \mathcal{B}\left(\overline{\mathbb{R}}^{d} \backslash\{\mathbf{0}\}\right)$. Note that $\mu_{s}(A) \leq \mu_{t}(A)$ for every $A$ if $s<t$ and $s, t \in U_{T}$, due to the independence of the increments (see, e.g. [9, Lemma 12]), and that, by the homogeneity property of the measures $\mu_{t}$, we have $\tilde{\mu}(S \times u A)=u^{-\alpha} \tilde{\mu}(S \times A)$ for every $S \in \mathscr{B}([0, T]), A \in \mathscr{B}\left(\overline{\mathbb{R}}^{d} \backslash\{\mathbf{0}\}\right)$, and $u>0$. The measure $\tilde{\mu}$ can be interpreted as the limit of the rescaled Lévy measure $\tilde{v}$ of $\boldsymbol{X}$, in the following way. Let $\left(\tilde{v}^{(u)}\right)_{u>0}$ be the collection of measures on $\mathcal{B}\left([0, T] \times \overline{\mathbb{R}}^{d} \backslash\{\mathbf{0}\}\right)$ determined by $\tilde{v}^{(u)}([0, t] \times A)=u^{\alpha} L(u) \tilde{v}([0, t] \times u A)$ for each $t \in[0, T]$ and $A \in \mathscr{B}\left(\overline{\mathbb{R}}^{d} \backslash\{\boldsymbol{0}\}\right)$.

Proposition 4.2. Let $(\boldsymbol{X})_{t \in[0, T]}$ be an $\mathbb{R}^{d}$-valued additive process satisfying

$$
\boldsymbol{X}_{T} \in \mathrm{RV}\left(\alpha, L, \mu_{T}\right) .
$$

If $u^{\alpha} L(u) \mathrm{P}\left(u^{-1} \boldsymbol{X}_{t} \in \cdot\right) \stackrel{\mathrm{v}}{\rightarrow} \mu_{t}(\cdot)$ on $\mathcal{B}\left(\overline{\mathbb{R}}^{d} \backslash\{\boldsymbol{0}\}\right)$ as $u \rightarrow \infty$, for all but at most countably many $t \in[0, T]$, then

$$
\tilde{\nu}^{(u)}(\cdot) \stackrel{\mathrm{v}}{\rightarrow} \tilde{\mu}(\cdot) \quad \text { on } \mathcal{B}\left([0, T] \times \overline{\mathbb{R}}^{d} \backslash\{\mathbf{0}\}\right) \text { as } u \rightarrow \infty .
$$


If $\boldsymbol{X}$ is a Lévy process then $\tilde{\boldsymbol{v}}(S \times A)=\operatorname{Leb}(S) v_{1}(A)$, where Leb denotes Lebesgue measure. Moreover, $\boldsymbol{X}_{T} \in \mathrm{RV}\left(\alpha, L, T \mu_{1}\right)$ implies that $\boldsymbol{X}_{t} \in \mathrm{RV}\left(\alpha, L, t \mu_{1}\right)$ for every $t \in[0, T]$; hence, $\tilde{\mu}(S \times A)=\operatorname{Leb}(S) \mu_{1}(A)$. In particular, $\tilde{\mu}(\{t\} \times A)=0$ for every $t \in[0, T]$.

Since additive processes are stochastically continuous (see [21, p. 3]) we always have $\tilde{v}(\{t\} \times u A)=0$ for every $t \in[0, T]$. However, for a general additive process, $\left(\tilde{v}^{(u)}\right)_{u>0}$ may have a degenerate vague limit as $u \rightarrow \infty$. One example is the additive process in Example 4.1, for which $\mu_{t}$ is the null measure for every $t<T$. In that example $\tilde{\mu}(S \times A)=\delta_{T}(S) \mu_{T}(A)$.

In connection with Proposition 4.2, we want to point out that we can construct an additive process on [0,1], say, for which $\boldsymbol{X}_{1}$ is regularly varying but $\left(u^{\alpha} L(u) \mathrm{P}\left(u^{-1} \boldsymbol{X}_{t} \in \cdot\right)\right)_{u>0}$ does not have a vague limit as $u \rightarrow \infty$, for any $t \in(0,1)$.

Example 4.2. Let $v$ and $\hat{v}$ be two Lévy measures on $\mathcal{B}\left(\mathbb{R}^{d} \backslash\{\boldsymbol{0}\}\right)$ with $v(A) \geq \hat{v}(A)$ for all $A \in \mathscr{B}\left(\mathbb{R}^{d} \backslash\{\boldsymbol{0}\}\right)$. Suppose that $v \in \mathrm{RV}(\alpha, L, \mu)$ but that $\left(u^{\alpha} L(u) \hat{v}(u \cdot)\right)_{u>0}$ does not converge vaguely as $u \rightarrow \infty$. Let the measure $\tilde{v}$ on $\mathcal{B}\left([0,1] \times \mathbb{R}^{d} \backslash\{\boldsymbol{0}\}\right)$ be given by

$$
\tilde{v}([0, t] \times A)=t(1-t) \hat{v}(A)+t v(A) .
$$

Furthermore, let $\xi$ be a Poisson random measure with intensity measure $\tilde{\nu}$. Then

$$
\boldsymbol{X}_{t}=\int_{[0, t] \times \mathbb{R}^{d} \backslash\{\mathbf{0}\}} \boldsymbol{x} \xi(\mathrm{d}(t, \boldsymbol{x})), \quad t \in[0,1],
$$

is an additive process and $\boldsymbol{X}_{t}$ has Lévy measure $t(1-t) \hat{v}+t v$. It follows from Proposition 3.1 that $\boldsymbol{X}_{1} \in \mathrm{RV}(\alpha, L, \mu)$ but that $\left(u^{\alpha} L(u) \mathrm{P}\left(u^{-1} \boldsymbol{X}_{t} \in \cdot\right)\right)_{u>0}$ does not converge vaguely as $u \rightarrow \infty$ for $t \in(0,1)$.

As already mentioned, the intuition behind Proposition 4.1 is that the process hits $u A$ during $[0, T]$ by making one big jump to this set. It is easy to determine the decay of the probability that the process makes a jump in $u A$, i.e. $\boldsymbol{X}_{t}-\boldsymbol{X}_{t-} \in u A$, for some $t \in[0, T]$. Consider an additive process $\boldsymbol{X}$ with $\boldsymbol{X}_{T} \in \mathrm{RV}\left(\alpha, L, \mu_{T}\right)$ and let $A \in \mathscr{B}\left(\mathbb{R}^{d}\right)$ be a set satisfying the conditions of Proposition 4.1. Then, with $\Delta \boldsymbol{X}_{t}=\boldsymbol{X}_{t}-\boldsymbol{X}_{t-}$,

$$
\begin{aligned}
\lim _{u \rightarrow \infty} u^{\alpha} L(u) \mathrm{P}\left(\Delta \boldsymbol{X}_{t} \in u A \text { for some } t \in[0, T]\right) & =\lim _{u \rightarrow \infty} u^{\alpha} L(u) \mathrm{P}(\xi([0, T] \times u A)>0) \\
& =\lim _{u \rightarrow \infty} u^{\alpha} L(u)(1-\exp \{-\tilde{v}([0, T] \times u A)\}) \\
& =\lim _{u \rightarrow \infty} u^{\alpha} L(u) v_{T}(u A) \\
& =\mu_{T}(A) .
\end{aligned}
$$

Under the conditions of Proposition 4.2 we can even find the asymptotics of the probability of occurrence of a big jump at some time $t$ within an arbitrary set $S \in \mathcal{B}([0, T])$ with $\tilde{\mu}(\partial(S \times$ A)) $=0$. Indeed,

$$
\begin{aligned}
\lim _{u \rightarrow \infty} u^{\alpha} L(u) \mathrm{P}\left(\Delta \boldsymbol{X}_{t} \in u A \text { for some } t \in S\right) & =\lim _{u \rightarrow \infty} u^{\alpha} L(u) \mathrm{P}(\xi(S \times u A)>0) \\
& =\lim _{u \rightarrow \infty} u^{\alpha} L(u)(1-\exp \{-\tilde{v}(S \times u A)\}) \\
& =\lim _{u \rightarrow \infty} u^{\alpha} L(u) \tilde{v}(S \times u A) \\
& =\tilde{\mu}(S \times A) .
\end{aligned}
$$

We finish with a remark that relates the result of Proposition 4.2 to regular variation on the space $\mathbb{D}$ of càdlàg functions equipped with the Skorokhod $J_{1}$ topology $[3$, p. 111]. 
Remark 4.2. If the additive process $\left(\boldsymbol{X}_{t}\right)_{t \in[0,1]}$ is regularly varying on the space of càdlàg functions (see [9]), such that $u^{\alpha} L(u) \mathrm{P}\left(u^{-1} \boldsymbol{X} \in B\right) \rightarrow m(B)$ as $u \rightarrow \infty$, for every $B \in \mathscr{B}(\mathbb{D})$ bounded away from $\mathbf{0}$ with $m(\partial B)=0$, then the conditions of Proposition 4.2 hold and the limit measure $m$ is given by

$$
m(B)=\int_{[0,1] \times \mathbb{R}^{d} \backslash\{\mathbf{0}\}} 1_{B}\left(\boldsymbol{y} 1_{[t, 1]}\right) \tilde{\mu}(\mathrm{d}(t, \boldsymbol{y})) .
$$

This representation is useful in determining the tail behavior of functionals of sample paths of the process using the continuous mapping theorem (cf. [9]).

\section{Proofs}

We denote by $B_{\mathbf{0}, r}=\left\{\boldsymbol{x} \in \mathbb{R}^{d}:|\boldsymbol{x}|<r\right\}$ the open ball of radius $r$ centered at $\mathbf{0}$. For a set $B$ we denote by $B^{\mathrm{c}}$ its complement. We also adopt the notation $\sum_{k=1}^{0}=0$.

\subsection{Proof of Proposition 3.1}

Since $\boldsymbol{X}$ is infinitely divisible, there exists a Lévy process $\left(\boldsymbol{X}_{t}\right)$ such that $\boldsymbol{X} \stackrel{\mathrm{D}}{=} \boldsymbol{X}_{1}$. The Lévy-Itô decomposition (see [21, Theorem 19.2, p. 120]) implies that $\boldsymbol{X}$ has representation $\boldsymbol{X} \stackrel{\mathrm{D}}{=} \tilde{\boldsymbol{X}}_{1}+\boldsymbol{J}$, where $\tilde{\boldsymbol{X}}_{1}$ and $\boldsymbol{J}$ are independent, $\left(\tilde{\boldsymbol{X}}_{t}\right)$ is a Lévy process with bounded jumps, and $\boldsymbol{J}$ has a compound Poisson distribution with representation $\boldsymbol{J} \stackrel{\mathrm{D}}{=} \sum_{k=1}^{N} \boldsymbol{Z}_{k}$, where $N$ is $\operatorname{Po}\left(v\left(B_{\mathbf{0}, 1}^{\mathrm{c}}\right)\right)$-distributed, $\mathrm{P}\left(\boldsymbol{Z}_{k} \in \cdot\right)=v\left(\cdot \cap \boldsymbol{B}_{\mathbf{0}, 1}^{\mathrm{c}}\right) / v\left(\boldsymbol{B}_{\mathbf{0}, 1}^{\mathrm{c}}\right)$, and $N, \boldsymbol{Z}_{1}, \boldsymbol{Z}_{2}, \ldots$ are independent. Note that ' $\stackrel{\text { D, }}{=}$ denotes equality in distribution. Since $\left(\tilde{\boldsymbol{X}}_{t}\right)$ has bounded jumps, it follows from Theorem 34 of [16, p. 25] that $\tilde{\boldsymbol{X}}_{1}$ has finite moments of all orders. Therefore, $\tilde{\boldsymbol{X}}_{1}$ will not contribute to the tail behavior of $\boldsymbol{X}_{t}$.

(i) First assume that $X \in \mathrm{RV}(\alpha, L, \mu)$. We will show that

$$
\begin{aligned}
X \in \mathrm{RV}(\alpha, L, \mu) & \stackrel{(\mathrm{a})}{\Longrightarrow} \boldsymbol{J} \in \mathrm{RV}(\alpha, L, \mu) \\
& \stackrel{(\mathrm{b})}{\Longrightarrow} Z_{1} \in \operatorname{RV}\left(\alpha, L, \mu / v\left(B_{\mathbf{0}, 1}^{\mathrm{c}}\right)\right) \\
& \stackrel{(\mathrm{c})}{\Longrightarrow} v \in \operatorname{RV}(\alpha, L, \mu) .
\end{aligned}
$$

To show implication (a), we first show that $\left(u^{\alpha} L(u) \mathrm{P}\left(u^{-1} \boldsymbol{J} \in \cdot\right)\right)_{u>0}$ is relatively compact in the vague topology. By Theorem 15.7 .5 of [13, p. 170], this is equivalent to showing that $\left.\lim \sup _{u \rightarrow \infty} u^{\alpha} L(u) \mathrm{P}\left(u^{-1} \boldsymbol{J} \in B_{\mathbf{0}, r}^{\mathrm{c}}\right)\right)<\infty$ for every $r>0$. Choose an $r>0$. We have

$$
\begin{aligned}
\limsup _{u \rightarrow \infty} u^{\alpha} L(u) \mathrm{P}\left(u^{-1} \boldsymbol{X} \in B_{\mathbf{0}, r / 2}^{\mathrm{c}}\right) & =\limsup _{u \rightarrow \infty} u^{\alpha} L(u) \mathrm{P}\left(u^{-1}\left(\tilde{\boldsymbol{X}}_{1}+\boldsymbol{J}\right) \in B_{\mathbf{0}, r / 2}^{\mathrm{c}}\right) \\
& \geq \limsup _{u \rightarrow \infty} u^{\alpha} L(u) \mathrm{P}\left(u^{-1} \boldsymbol{J} \in B_{\mathbf{0}, r}^{\mathrm{c}}\right) \mathrm{P}\left(u^{-1} \tilde{\boldsymbol{X}}_{1} \in B_{\mathbf{0}, r / 2}\right) \\
& =\limsup _{u \rightarrow \infty} u^{\alpha} L(u) \mathrm{P}\left(u^{-1} \boldsymbol{J} \in B_{\mathbf{0}, r}^{\mathrm{c}}\right) .
\end{aligned}
$$

We conclude that $\left(u^{\alpha} L(u) \mathrm{P}\left(u^{-1} \boldsymbol{J} \in \cdot\right)\right)_{u>0}$ is relatively compact. Let $\mu_{1}$ be a subsequential vague limit such that $u_{i}^{\alpha} L\left(u_{i}\right) \mathrm{P}\left(u_{i}{ }^{-1} \boldsymbol{J} \in \cdot\right) \stackrel{\mathrm{v}}{\rightarrow} \mu_{1}(\cdot)$ as $i \rightarrow \infty$ for some sequence $\left(u_{i}\right)$ with $\lim _{i \rightarrow \infty} u_{i}=\infty$. Since $\tilde{\boldsymbol{X}}_{1}$ has finite moments of all orders, for every $\varepsilon>0$ we have

$$
u_{i}^{\alpha} L\left(u_{i}\right) \mathrm{P}\left(\left|\tilde{\boldsymbol{X}}_{1}\right|>\varepsilon u_{i}\right) \leq \frac{u_{i}^{\alpha} L\left(u_{i}\right) \mathrm{E}\left(\left|\tilde{\boldsymbol{X}}_{1}\right|^{2 \alpha}\right)}{\left(\varepsilon u_{i}\right)^{2 \alpha}} \rightarrow 0 \quad \text { as } i \rightarrow \infty .
$$


Hence, $u_{i}^{\alpha} L\left(u_{i}\right) \mathrm{P}\left(u_{i}{ }^{-1} \tilde{\boldsymbol{X}}_{1} \in \cdot\right) \stackrel{\mathrm{v}}{\rightarrow} 0$ (the zero measure on $\left.\mathcal{B}\left(\overline{\mathbb{R}}^{d} \backslash\{\boldsymbol{0}\}\right)\right)$ as $i \rightarrow \infty$, so by Proposition A.1(i), below, $u_{i}^{\alpha} L\left(u_{i}\right) \mathrm{P}\left(u_{i}^{-1} \boldsymbol{X} \in \cdot\right) \stackrel{\mathrm{v}}{\rightarrow} \mu_{1}(\cdot)$ as $i \rightarrow \infty$. However, we have assumed that $\boldsymbol{X} \in \mathrm{RV}(\alpha, L, \mu)$, so $\mu_{1}=\mu$. Hence, implication (a) holds.

Implication (b) follows directly from Proposition A.2(ii), below.

Implication (c) follows since $\mathrm{P}\left(\boldsymbol{Z}_{k} \in \cdot\right)=v\left(\cdot \cap B_{\mathbf{0}, 1}^{\mathrm{c}}\right) / v\left(B_{\mathbf{0}, 1}^{\mathrm{c}}\right)$ and, for any relatively compact set $B \in \mathscr{B}\left(\overline{\mathbb{R}}^{d} \backslash\{\mathbf{0}\}\right)$ and sufficiently large $u, v\left((u B) \cap B_{\mathbf{0}, 1}^{\mathrm{c}}\right)=v(u B)$.

(ii) Now assume that $v \in \mathrm{RV}(\alpha, L, \mu)$. Then, since $\mathrm{P}\left(\boldsymbol{Z}_{k} \in \cdot\right)=v\left(\cdot \cap B_{\mathbf{0}, 1}^{\mathrm{c}}\right) / v\left(B_{\mathbf{0}, 1}^{\mathrm{c}}\right)$, it follows that $\boldsymbol{Z}_{k} \in \operatorname{RV}\left(\alpha, L, \mu / v\left(B_{\mathbf{0}, 1}^{\mathrm{c}}\right)\right)$, because $v\left((u B) \cap B_{\mathbf{0}, 1}^{\mathrm{c}}\right)=v(u B)$ for any relatively compact set $B \in \mathcal{B}\left(\overline{\mathbb{R}}^{d} \backslash\{\mathbf{0}\}\right)$ and sufficiently large $u$. Furthermore, by Proposition A.2(i), we have $\boldsymbol{J} \in \operatorname{RV}\left(\alpha, L, \mathrm{E}(N) \mu / \nu\left(B_{\mathbf{0}, 1}^{\mathrm{c}}\right)\right)$ and $\mathrm{E}(N) \mu / \nu\left(B_{\mathbf{0}, 1}^{\mathrm{c}}\right)=\mu$. Since $\boldsymbol{X} \stackrel{\mathrm{D}}{=} \tilde{\boldsymbol{X}}_{1}+\boldsymbol{J}$ (where $\tilde{\boldsymbol{X}}_{1}$ and $\boldsymbol{J}$ are independent), $\tilde{\boldsymbol{X}}_{1}$ has finite moments of all orders, and $\boldsymbol{J} \in \operatorname{RV}(\alpha, L, \mu)$, the conclusion follows from Proposition A.1(i).

\subsection{Proof of Proposition 4.1}

By assumption, $\lim _{u \rightarrow \infty} u^{\alpha} L(u) \mathrm{P}\left(\boldsymbol{X}_{T} \in u A\right)=\mu_{T}(A)>0$. Hence, $\mathrm{P}\left(\boldsymbol{X}_{T} \in u A\right)>0$ for sufficiently large $u$. Since $\mathrm{P}\left(\boldsymbol{X}_{t} \in u A\right.$ for some $\left.t \in[0, T]\right) \geq \mathrm{P}\left(\boldsymbol{X}_{T} \in u A\right)$ we need only show that

$$
\limsup _{u \rightarrow \infty} \frac{\mathrm{P}\left(\boldsymbol{X}_{t} \in u A \text { for some } t \in[0, T]\right)}{\mathrm{P}\left(\boldsymbol{X}_{T} \in u A\right)} \leq 1 .
$$

Set $A^{\varepsilon}=\left\{\boldsymbol{y} \in \mathbb{R}^{d}:|\boldsymbol{y}-\boldsymbol{x}| \leq \varepsilon, \boldsymbol{x} \in A\right\}$ with $\varepsilon>0$ small enough that $\mathbf{0} \notin A^{\varepsilon}$. Following $[9$, p. 265], denote by $B(p, \varepsilon,[0, T])$ the set of càdlàg functions that $\varepsilon$-oscillate at least $p$ times in $[0, T]$. That is, for $\boldsymbol{x} \in B(p, \varepsilon,[0, T])$ there exist $t_{0}, t_{1}, \ldots, t_{p}, 0 \leq t_{0}<t_{1}<\cdots<t_{p} \leq T$, with $\left|\boldsymbol{x}_{t_{i}}-\boldsymbol{x}_{t_{i-1}}\right|>\varepsilon, i=1, \ldots, p$. We have

$$
\begin{aligned}
\mathrm{P}\left(\boldsymbol{X}_{t} \in\right. & u A \text { for some } t \in[0, T]) \\
= & \mathrm{P}\left(\boldsymbol{X}_{t} \in u A \text { for some } t \in[0, T], \boldsymbol{X} \in u B(2, \varepsilon,[0, T])\right) \\
& +\mathrm{P}\left(\boldsymbol{X}_{t} \in u A \text { for some } t \in[0, T], \boldsymbol{X} \notin u B(2, \varepsilon,[0, T])\right) \\
\leq & \mathrm{P}(\boldsymbol{X} \in u B(2, \varepsilon,[0, T]))+\mathrm{P}\left(\boldsymbol{X}_{T} \in u A^{\varepsilon}\right) .
\end{aligned}
$$

Since $\left(\boldsymbol{X}_{t}\right)_{t \in[0, T]}$ is a strong Markov process satisfying the conditions of Lemma 21 of [9] (also see Theorem 2.5 and Lemma 2.8 of [14]), we have

$$
\limsup _{u \rightarrow \infty} u^{\alpha} L(u) \mathrm{P}(\boldsymbol{X} \in u B(2, \varepsilon,[0, T]))=0 .
$$

The Portmanteau theorem (see [13, Theorem 15.7.2, p. 169]) yields

$$
\limsup _{u \rightarrow \infty} u^{\alpha} L(u) \mathrm{P}\left(\boldsymbol{X}_{T} \in u A^{\varepsilon}\right) \leq \mu_{T}\left(A^{\varepsilon}\right) .
$$

Since $\mu_{T}\left(A^{\varepsilon}\right) \downarrow \mu_{T}(\bar{A})=\mu_{T}(A)$ as $\varepsilon \downarrow 0$, we find that

$$
\limsup _{u \rightarrow \infty} u^{\alpha} L(u) \mathrm{P}\left(\boldsymbol{X}_{t} \in u A \text { for some } t \in[0, T]\right) \leq \mu_{T}(A) .
$$

Hence,

$$
\begin{aligned}
\limsup _{u \rightarrow \infty} & \frac{\mathrm{P}\left(\boldsymbol{X}_{t} \in u A \text { for some } t \in[0, T]\right)}{\mathrm{P}\left(\boldsymbol{X}_{T} \in u A\right)} \\
= & \limsup _{u \rightarrow \infty} \frac{u^{\alpha} L(u) \mathrm{P}\left(\boldsymbol{X}_{t} \in u A \text { for some } t \in[0, T]\right)}{u^{\alpha} L(u) \mathrm{P}\left(\boldsymbol{X}_{T} \in u A\right)} \leq 1 .
\end{aligned}
$$




\subsection{Proof of Proposition 4.2}

Let $U_{T} \subset[0, T]$ contain those $t \in[0, T]$ for which $u^{\alpha} L(u) \mathrm{P}\left(u^{-1} \boldsymbol{X}_{t} \in \cdot\right) \stackrel{\mathrm{v}}{\rightarrow} \mu_{t}(\cdot)$. The proof consists of two steps. First we show that

(a) for every $t \in U_{T}, u^{\alpha} L(u) v_{t}(u \cdot) \stackrel{\mathrm{v}}{\rightarrow} \mu_{t}(\cdot)$ on $\mathscr{B}\left(\overline{\mathbb{R}}^{d} \backslash\{\mathbf{0}\}\right)$ as $u \rightarrow \infty$.

Then we construct a convergence-determining class $\mathcal{C}$ for vague convergence to $\tilde{\mu}$, and show that

(b) for every $C \in \mathcal{C}, \tilde{v}^{(u)}(C) \rightarrow \tilde{\mu}(C)$ as $u \rightarrow \infty$.

If $\mu_{t}$ is nonzero then (a) follows from Proposition 3.1. Suppose that $\mu_{t}=0$. To avoid trivialities we may assume that $v_{t}$ has unbounded support. By the Lévy-Itô decomposition, we have $\boldsymbol{X}_{t} \stackrel{\mathrm{D}}{=} \tilde{\boldsymbol{X}}_{t}+\sum_{k=1}^{N_{t}} \boldsymbol{Z}_{k}$, where the $\boldsymbol{Z}_{k}$ are mutually independent, identically distributed, and independent of $N_{t}$, with

$$
\mathrm{P}\left(\boldsymbol{Z}_{1} \in \cdot\right)=v_{t}\left(\cdot \cap B_{\mathbf{0}, 1}^{\mathrm{c}}\right) / v_{t}\left(B_{\mathbf{0}, 1}^{\mathrm{c}}\right) .
$$

For every $r>0$, we have

$$
\begin{aligned}
0 & =\limsup _{u \rightarrow \infty} u^{\alpha} L(u) \mathrm{P}\left(u^{-1} \boldsymbol{X}_{t} \in B_{\mathbf{0}, r / 2}^{\mathrm{c}}\right) \\
& \geq \limsup _{u \rightarrow \infty} u^{\alpha} L(u) \mathrm{P}\left(u^{-1} \sum_{k=1}^{N_{t}} \boldsymbol{Z}_{k} \in B_{\mathbf{0}, r}^{\mathrm{c}}\right) \mathrm{P}\left(u^{-1} \tilde{\boldsymbol{X}}_{t} \in B_{\mathbf{0}, r / 2}\right) \\
& =\limsup _{u \rightarrow \infty} u^{\alpha} L(u) \mathrm{P}\left(u^{-1} \sum_{k=1}^{N_{t}} \boldsymbol{Z}_{k} \in B_{\mathbf{0}, r}^{\mathrm{c}}\right) \\
& \geq \limsup _{u \rightarrow \infty} u^{\alpha} L(u) \mathrm{P}\left(u^{-1} \boldsymbol{Z}_{1} \in B_{\mathbf{0}, 2 r}^{\mathrm{c}}, u^{-1} \sum_{k=2}^{N_{t}} \boldsymbol{Z}_{k} \in B_{\mathbf{0}, r}\right) \\
& =\limsup _{u \rightarrow \infty} u^{\alpha} L(u) \mathrm{P}\left(u^{-1} \boldsymbol{Z}_{1} \in B_{\mathbf{0}, 2 r}^{\mathrm{c}}\right) \\
& =\limsup _{u \rightarrow \infty} u^{\alpha} L(u) v_{t}\left(u B_{\mathbf{0}, 2 r}^{\mathrm{c}}\right) .
\end{aligned}
$$

Hence, $u^{\alpha} L(u) v_{t}(u \cdot) \stackrel{\mathrm{v}}{\rightarrow} 0$ as $u \rightarrow \infty$. This completes the proof of part (a).

Now consider part (b). First we have to find an appropriate convergence-determining class C. If $W_{T}$ is a subset of $(0, T)$ with the property that its complement is at most countable, then a convergence-determining class is given by

$$
\mathcal{C}=\left\{[s, t] \times A: s, t \in W_{T} \cup\{0, T\}, A \in \mathcal{B}\left(\overline{\mathbb{R}}^{d} \backslash\{\boldsymbol{0}\}\right) \text { is relatively compact, } \mu_{T}(\partial A)=0\right\} .
$$

To specify $W_{T}$, let

$$
V_{T}=\left\{t \in(0, T): \tilde{\mu}\left(\{t\} \times B_{\mathbf{0}, 1}^{\mathrm{c}}\right)=0\right\} .
$$

Since $\tilde{\mu}\left([0, T] \times B_{\mathbf{0}, 1}^{\mathrm{c}}\right)<\infty$, it follows that the complement of $V_{T}$ is at most countable. Now let $W_{T}=U_{T} \cap V_{T}$. We note that, for every relatively compact set $A \in \mathscr{B}\left(\overline{\mathbb{R}}^{d} \backslash\{\boldsymbol{0}\}\right)$, there exists an $r>0$ such that $A \subset B_{\mathbf{0}, r}^{\mathrm{c}}$, and by the homogeneity property of $\tilde{\mu}$ we have

$$
\tilde{\mu}(\{t\} \times A) \leq \tilde{\mu}\left(\{t\} \times B_{\mathbf{0}, r}^{\mathrm{c}}\right)=r^{-\alpha} \tilde{\mu}\left(\{t\} \times B_{\mathbf{0}, 1}^{\mathrm{c}}\right)=0 \quad \text { for every } t \in V_{T} .
$$


Finally, we show convergence of sets in $\mathcal{C}$. Let $[s, t] \times A \in \mathcal{C}$. Notice that, since $\mu_{T}(\partial A)=0$, it follows by construction that $\mu_{t}(\partial A) \leq \mu_{T}(\partial A)=0$ for $t<T$. Since $\tilde{v}(\{s\} \times u A)=0$, we have

$$
\begin{aligned}
\tilde{v}^{(u)}([s, t] \times A) & =u^{\alpha} L(u) \tilde{v}([0, t] \times u A)-u^{\alpha} L(u) \tilde{v}([0, s] \times u A) \\
& \rightarrow \tilde{\mu}([0, t] \times A)-\tilde{\mu}([0, s] \times A)
\end{aligned}
$$

as $u \rightarrow \infty$. Since $s \in V_{T}$ implies that $\tilde{\mu}(\{s\} \times A)=0$, we conclude that

$$
\tilde{v}^{(u)}([s, t] \times A) \rightarrow \tilde{\mu}([s, t] \times A)
$$

as $u \rightarrow \infty$. We complete the proof by showing that (b) implies that $\tilde{v}^{(u)} \stackrel{\mathrm{v}}{\rightarrow} \tilde{\mu}$ on $\mathscr{B}([0, T] \times$ $\left.\overline{\mathbb{R}}^{d} \backslash\{\boldsymbol{0}\}\right)$ as $u \rightarrow \infty$. For every relatively compact set $B \in \mathscr{B}\left([0, T] \times \overline{\mathbb{R}}^{d} \backslash\{\boldsymbol{0}\}\right)$, there exists an $r>0$ such that

$$
\begin{aligned}
\limsup _{u \rightarrow \infty} \tilde{v}^{(u)}(B) & \leq \limsup _{u \rightarrow \infty} \tilde{v}^{(u)}\left([0, T] \times B_{\mathbf{0}, r}^{\mathrm{c}}\right) \\
& =\limsup _{u \rightarrow \infty} u^{\alpha} L(u) \nu_{T}\left(u B_{\mathbf{0}, r}^{\mathrm{c}}\right) \\
& =\mu_{T}\left(B_{\mathbf{0}, r}^{\mathrm{c}}\right) \\
& <\infty
\end{aligned}
$$

It follows that $\left(\tilde{v}^{(u)}\right)_{u>0}$ is vaguely relatively compact. Moreover, we have shown that any two subsequential vague limits must agree on the $\pi$-system $\mathcal{C}$. Since $\mathcal{C}$ generates the $\sigma$-algebra $\mathscr{B}\left([0, T] \times \overline{\mathbb{R}}^{d} \backslash\{\boldsymbol{0}\}\right)$, applying Theorem 10.3 of $[4$, p. 163] completes the proof.

\section{Appendix A. Sums of regularly varying random vectors}

In this section we will derive some useful results concerning sums of regularly varying random vectors.

Proposition A.1. Let $\boldsymbol{X}$ be an $\mathbb{R}^{d}$-valued random vector in $\mathrm{RV}(\alpha, L, \mu)$.

(i) Let $\tilde{\boldsymbol{X}}$ be an $\mathbb{R}^{d}$-valued random vector independent of $\boldsymbol{X}$. If $\tilde{\boldsymbol{X}}$ satisfies

$$
u^{\alpha} L(u) \mathrm{P}\left(u^{-1} \tilde{\boldsymbol{X}} \in \cdot\right) \stackrel{\mathrm{v}}{\rightarrow} \tilde{\mu}(\cdot)
$$

on $\mathcal{B}\left(\overline{\mathbb{R}}^{d} \backslash\{\mathbf{0}\}\right)$ as $u \rightarrow \infty$, for some Radon measure $\tilde{\mu}$ (possibly the null measure) with $\tilde{\mu}\left(\overline{\mathbb{R}}^{d} \backslash \mathbb{R}^{d}\right)=0$, then $\boldsymbol{X}+\tilde{\boldsymbol{X}} \in \operatorname{RV}(\alpha, L, \mu+\tilde{\mu})$.

(ii) If, for some $k \geq 1$, there exist independent, identically distributed random vectors $\boldsymbol{X}_{i}$, $i=1, \ldots, k$, such that $\boldsymbol{X} \stackrel{\mathrm{D}}{=} \boldsymbol{X}_{1}+\cdots+\boldsymbol{X}_{k}$, then $\boldsymbol{X}_{i} \in \mathrm{RV}(\alpha, L, \mu / k)$ for $i=1, \ldots, k$.

Remark A.1. A statement similar to (i), but for $\mathbb{R}_{+}^{d}$-valued random vectors, was proved in Proposition 4.1 of [18].

Proof of Proposition A.1. (i) Let $\varepsilon>0$ and note that, by Remark 2.1(i), $\mu\left(\partial B_{\mathbf{0}, \varepsilon}\right)=$ $\tilde{\mu}\left(\partial B_{0, \varepsilon}\right)=0$. Since

$$
u^{\alpha} L(u) \mathrm{P}\left(u^{-1}(\boldsymbol{X}, \tilde{\boldsymbol{X}}) \in B_{\mathbf{0}, \varepsilon}^{\mathrm{c}} \times B_{\mathbf{0}, \varepsilon}^{\mathrm{c}}\right)=u^{\alpha} L(u) \mathrm{P}\left(u^{-1} \boldsymbol{X} \in B_{\mathbf{0}, \varepsilon}^{\mathrm{c}}\right) \mathrm{P}\left(u^{-1} \tilde{\boldsymbol{X}} \in B_{\mathbf{0}, \varepsilon}^{\mathrm{c}}\right) \rightarrow 0
$$

as $u \rightarrow \infty$, it follows that $(\boldsymbol{X}, \tilde{\boldsymbol{X}}) \in \operatorname{RV}(\alpha, L, \hat{\mu})$, where $\hat{\mu}$ is a Radon measure on $\mathcal{B}\left(\overline{\mathbb{R}}^{2 d} \backslash\{\boldsymbol{0}\}\right)$ that concentrates on $\left(\{\mathbf{0}\} \times \mathbb{R}^{d}\right) \cup\left(\mathbb{R}^{d} \times\{\mathbf{0}\}\right)$. Let $T: \mathbb{R}^{2 d} \rightarrow \mathbb{R}^{d}$ be the linear transformation 
$T(\boldsymbol{x}, \tilde{\boldsymbol{x}})=\boldsymbol{x}+\tilde{\boldsymbol{x}}$. By Proposition 3.18 of [19, p. 148], $\boldsymbol{X}+\tilde{\boldsymbol{X}} \in \mathrm{RV}\left(\alpha, L, \hat{\mu} \circ T^{-1}\left(\cdot \cap \mathbb{R}^{d}\right)\right)$. Moreover, for any $B \in \mathscr{B}\left(\overline{\mathbb{R}}^{d} \backslash\{\mathbf{0}\}\right)$,

$$
\begin{aligned}
\hat{\mu} \circ T^{-1}\left(B \cap \mathbb{R}^{d}\right) & =\hat{\mu}\left(\left\{(\boldsymbol{x}, \tilde{\boldsymbol{x}}): \boldsymbol{x}+\tilde{\boldsymbol{x}} \in B \cap \mathbb{R}^{d}\right\}\right) \\
& =\hat{\mu}\left(\left\{(\boldsymbol{x}, \mathbf{0}): \boldsymbol{x}+\mathbf{0} \in B \cap \mathbb{R}^{d}\right\}\right)+\hat{\mu}\left(\left\{(\mathbf{0}, \tilde{\boldsymbol{x}}): \mathbf{0}+\tilde{\boldsymbol{x}} \in B \cap \mathbb{R}^{d}\right\}\right) \\
& =\mu(B)+\tilde{\mu}(B) .
\end{aligned}
$$

(ii) Since $\boldsymbol{X} \in \mathrm{RV}(\alpha, L, \mu)$ it follows that, for any subsequence $\left(u_{j}\right)$ with $\lim _{j \rightarrow \infty} u_{j}=\infty$, we have $u_{j}^{\alpha} L\left(u_{j}\right) \mathrm{P}\left(u_{j}^{-1} \boldsymbol{X} \in \cdot\right) \stackrel{\mathrm{v}}{\rightarrow} \mu(\cdot)$ on $\mathscr{B}\left(\overline{\mathbb{R}}^{d} \backslash\{\mathbf{0}\}\right)$. Hence, it follows from part (i) that any subsequential vague limit $\mu_{1}$ of $\left(u^{\alpha} L(u) \mathrm{P}\left(u^{-1} \boldsymbol{X}_{1} \in \cdot\right)\right)_{u>0}$ must satisfy $\mu_{1}=\mu / k$. Hence, we need only show that $\left(u^{\alpha} L(u) \mathrm{P}\left(u^{-1} \boldsymbol{X}_{1} \in \cdot\right)\right)_{u>0}$ is relatively compact in the vague topology. By Theorem 15.7.5 of [13, p. 170], this is equivalent to showing that

$$
\sup _{u>0} u^{\alpha} L(u) \mathrm{P}\left(u^{-1} \boldsymbol{X}_{1} \in B\right)<\infty
$$

for every relatively compact set $B \in \mathcal{B}\left(\overline{\mathbb{R}}^{d} \backslash\{\boldsymbol{0}\}\right)$. In turn, this is equivalent to showing that $\lim \sup _{u \rightarrow \infty} u^{\alpha} L(u) \mathrm{P}\left(u^{-1} \boldsymbol{X}_{1} \in B_{\mathbf{0}, r}^{\mathrm{c}}\right)<\infty$ for every $r>0$. Let $r>0$ and $\varepsilon \in(0, r / k)$. Then

$$
\begin{aligned}
& \limsup _{u \rightarrow \infty} u^{\alpha} L(u) \mathrm{P}\left(u^{-1} \boldsymbol{X} \in B_{\mathbf{0}, r-k \varepsilon}^{\mathrm{c}}\right) \\
& \quad=\limsup _{u \rightarrow \infty} u^{\alpha} L(u) \mathrm{P}\left(u^{-1}\left(\boldsymbol{X}_{1}+\cdots+\boldsymbol{X}_{k}\right) \in B_{\mathbf{0}, r-k \varepsilon}^{\mathrm{c}}\right) \\
& \quad \geq \limsup _{u \rightarrow \infty} u^{\alpha} L(u) \mathrm{P}\left(u^{-1} \boldsymbol{X}_{1} \in B_{\mathbf{0}, r}^{\mathrm{c}}, u^{-1} \boldsymbol{X}_{j} \in B_{\mathbf{0}, \varepsilon} \text { for } j=2, \ldots, k\right) \\
& \quad=\limsup _{u \rightarrow \infty} u^{\alpha} L(u) \mathrm{P}\left(u^{-1} \boldsymbol{X}_{1} \in B_{\mathbf{0}, r}^{\mathrm{c}}\right) \mathrm{P}\left(u^{-1} \boldsymbol{X}_{1} \in B_{\mathbf{0}, \varepsilon}\right)^{k-1} \\
& \quad=\limsup _{u \rightarrow \infty} u^{\alpha} L(u) \mathrm{P}\left(u^{-1} \boldsymbol{X}_{1} \in B_{\mathbf{0}, r}^{\mathrm{c}}\right)
\end{aligned}
$$

Hence, $\lim \sup _{u \rightarrow \infty} u^{\alpha} L(u) \mathrm{P}\left(u^{-1} \boldsymbol{X}_{1} \in B_{\mathbf{0}, r}^{\mathrm{c}}\right)<\infty$, which shows the required relative compactness. This completes the proof.

Above we considered a sum of a deterministic number of terms. We will now consider the case with a random number of terms.

Proposition A.2. Let $\left(\boldsymbol{X}_{k}\right)_{k \geq 1}$ be a sequence of independent, identically distributed $\mathbb{R}^{d}$ valued random vectors, and let $N \geq 0$ be an integer-valued random variable satisfying $\sum_{n=1}^{\infty} \mathrm{P}(N=n)(1+\varepsilon)^{n}<\infty$ for some $\varepsilon>0$. Suppose that $N$ and $\left(\boldsymbol{X}_{k}\right)_{k \geq 1}$ are independent and that $N$ is not almost surely 0 .

(i) If $\boldsymbol{X}_{1} \in \mathrm{RV}(\alpha, L, \mu)$ then $\sum_{k=1}^{N} \boldsymbol{X}_{k} \in \mathrm{RV}(\alpha, L, \mathrm{E}(N) \mu)$.

(ii) If $\sum_{k=1}^{N} \boldsymbol{X}_{k} \in \mathrm{RV}(\alpha, L, \mu)$ then $\boldsymbol{X}_{1} \in \mathrm{RV}(\alpha, L, \mu / \mathrm{E}(N))$.

Proof. (i) Take a relatively compact set $B \in \mathcal{B}\left(\overline{\mathbb{R}}^{d} \backslash\{\boldsymbol{0}\}\right)$ with $\mu(\partial B)=0$. Since

$$
\mu\left(\overline{\mathbb{R}}^{d} \backslash \mathbb{R}^{d}\right)=0
$$


we may without loss of generality take $B \subset \mathbb{R}^{d}$. Furthermore, since $B \subset B_{\mathbf{0}, r}^{\mathrm{c}}$ for some $r>0$, since

$$
\begin{aligned}
u^{\alpha} L(u) \mathrm{P}\left(u^{-1} \sum_{k=1}^{N} \boldsymbol{X}_{k} \in B\right) & =\sum_{l=1}^{\infty} u^{\alpha} L(u) \mathrm{P}\left(u^{-1} \sum_{k=1}^{l} \boldsymbol{X}_{k} \in B\right) \mathrm{P}(N=l) \\
& \leq \sum_{l=1}^{\infty} u^{\alpha} L(u) \mathrm{P}\left(u^{-1} \sum_{k=1}^{l}\left|\boldsymbol{X}_{k}\right| \geq r\right) \mathrm{P}(N=l)
\end{aligned}
$$

and since the nonnegative random variables $\left|\boldsymbol{X}_{k}\right|$ are regularly varying, it follows from Theorem 3 of [7] that (A.1) converges to $\mathrm{E}(N) \mu\left(B_{0}^{\mathrm{c}}, r\right)$. Using Pratt's theorem (see [15]) and Proposition A.1(i), we conclude that we may interchange the order of the summation and the limit in the following, to obtain

$$
\begin{aligned}
\lim _{u \rightarrow \infty} u^{\alpha} L(u) \mathrm{P}\left(u^{-1} \sum_{k=1}^{N} \boldsymbol{X}_{k} \in B\right) & =\lim _{u \rightarrow \infty} \sum_{l=1}^{\infty} u^{\alpha} L(u) \mathrm{P}\left(u^{-1} \sum_{k=1}^{l} \boldsymbol{X}_{k} \in B\right) \mathrm{P}(N=l) \\
& =\sum_{l=1}^{\infty} \lim _{u \rightarrow \infty} u^{\alpha} L(u) \mathrm{P}\left(u^{-1} \sum_{k=1}^{l} \boldsymbol{X}_{k} \in B\right) \mathrm{P}(N=l) \\
& =\mathrm{E}(N) \mu(B) .
\end{aligned}
$$

(ii) We first show that $\left(u^{\alpha} L(u) \mathrm{P}\left(u^{-1} \boldsymbol{X}_{1} \in \cdot\right)\right)_{u>0}$ is vaguely relatively compact. Since, for every $r>0$,

$$
\begin{aligned}
& \limsup _{u \rightarrow \infty} u^{\alpha} L(u) \mathrm{P}\left(u^{-1} \boldsymbol{X}_{1} \in B_{\mathbf{0}, r}^{\mathrm{c}}\right) \\
& \quad=\limsup _{u \rightarrow \infty} u^{\alpha} L(u) \mathrm{P}\left(u^{-1} \boldsymbol{X}_{1} \in B_{\mathbf{0}, r}^{\mathrm{c}}\right) \mathrm{P}\left(u^{-1} \sum_{k=2}^{N} \boldsymbol{X}_{k} \in B_{\mathbf{0}, r / 2}\right) \\
& \quad=\limsup _{u \rightarrow \infty} u^{\alpha} L(u) \mathrm{P}\left(u^{-1} \boldsymbol{X}_{1} \in B_{\mathbf{0}, r}^{\mathrm{c}}, u^{-1} \sum_{k=2}^{N} \boldsymbol{X}_{k} \in B_{\mathbf{0}, r / 2}\right) \\
& \quad \leq \limsup _{u \rightarrow \infty} u^{\alpha} L(u) \mathrm{P}\left(u^{-1} \sum_{k=1}^{N} \boldsymbol{X}_{k} \in B_{\mathbf{0}, r / 2}^{\mathrm{c}}\right) \\
& \quad<\infty,
\end{aligned}
$$

it follows that $\limsup _{u \rightarrow \infty} u^{\alpha} L(u) \mathrm{P}\left(u^{-1} \boldsymbol{X}_{1} \in B\right)<\infty$ for every relatively compact set $B \in \mathcal{B}\left(\overline{\mathbb{R}}^{d} \backslash\{\boldsymbol{0}\}\right)$, which shows the required vague relative compactness. We will show that any two subsequential vague limits of $\left(u^{\alpha} L(u) \mathrm{P}\left(u^{-1} X_{1} \in \cdot\right)\right)_{u>0}$ coincide and are equal to $\mu / \mathrm{E}(N)$. Suppose that, as $j \rightarrow \infty, u_{j}^{\alpha} L\left(u_{j}\right) \mathrm{P}\left(u_{j}^{-1} \boldsymbol{X}_{1} \in \cdot\right) \stackrel{\mathrm{v}}{\rightarrow} \mu_{1}(\cdot)$ on $\mathscr{B}\left(\overline{\mathbb{R}}^{d} \backslash\{\boldsymbol{0}\}\right)$ for some Radon measure $\mu_{1}$ and some sequence $\left(u_{j}\right)$ with $\lim _{j \rightarrow \infty} u_{j}=\infty$. Choose a relatively compact set $B \in \mathcal{B}\left(\overline{\mathbb{R}}^{d} \backslash\{\boldsymbol{0}\}\right)$ with $\mu_{1}(\partial B)=\mu(\partial B)=0$. We have

$$
u_{j}^{\alpha} L\left(u_{j}\right) \mathrm{P}\left(u_{j}^{-1} \sum_{k=1}^{N} \boldsymbol{X}_{k} \in B\right)=\sum_{l=1}^{\infty} \underbrace{u_{j}^{\alpha} L\left(u_{j}\right) \mathrm{P}\left(u_{j}^{-1} \sum_{k=1}^{l} \boldsymbol{X}_{k} \in B\right) \mathrm{P}(N=l)}_{f_{j}(l)} \rightarrow \mu(B)
$$


as $j \rightarrow \infty$ and, for some $r>0$,

$$
\begin{aligned}
u_{j}^{\alpha} L\left(u_{j}\right) \mathrm{P}\left(u_{j}^{-1} \sum_{k=1}^{N} \boldsymbol{X}_{k} \in B\right) & \leq u_{j}^{\alpha} L\left(u_{j}\right) \mathrm{P}\left(u_{j}^{-1} \sum_{k=1}^{N} \boldsymbol{X}_{k} \in B_{\mathbf{0}, r}^{\mathrm{c}}\right) \\
& =\sum_{l=1}^{\infty} \underbrace{u_{j}^{\alpha} L\left(u_{j}\right) \mathrm{P}\left(u_{j}^{-1} \sum_{k=1}^{l} \boldsymbol{X}_{k} \in B_{\mathbf{0}, r}^{\mathrm{c}}\right) \mathrm{P}(N=l)}_{G_{j}(l)} \\
& \rightarrow \mu\left(B_{\mathbf{0}, r}^{\mathrm{c}}\right)
\end{aligned}
$$

as $j \rightarrow \infty$. By Proposition A.1(i) (the same proof applies in this case), $\lim _{j \rightarrow \infty} f_{j}(l)=$ $l \mathrm{P}(N=l) \mu_{1}(B)$ and $\lim _{j \rightarrow \infty} G_{j}(l)=l \mathrm{P}(N=l) \mu_{1}\left(B_{\mathbf{0}, r}^{\mathrm{c}}\right)$. Moreover, $f_{j} \leq G_{j}$ for every $j$ and, by assumption, $\lim _{j \rightarrow \infty} \sum_{l=1}^{\infty} G_{j}(l)=\mu\left(B_{\mathbf{0}, r}^{\mathrm{c}}\right)<\infty$. Hence, Pratt's theorem applies and yields $\lim _{j \rightarrow \infty} \sum_{l=1}^{\infty} f_{j}(l)=\mathrm{E}(N) \mu_{1}(B)$. However,

$$
\lim _{j \rightarrow \infty} \sum_{l=1}^{\infty} f_{j}(l)=\lim _{j \rightarrow \infty} u_{j}^{\alpha} L\left(u_{j}\right) \mathrm{P}\left(u_{j}^{-1} \sum_{k=1}^{N} \boldsymbol{X}_{k} \in B\right)=\mu(B) .
$$

Since the relatively compact sets $B \in \mathcal{B}\left(\overline{\mathbb{R}}^{d} \backslash\{\mathbf{0}\}\right)$ with $\mu_{1}(\partial B)=\mu(\partial B)=0$ determine a Radon measure on $\mathscr{B}\left(\overline{\mathbb{R}}^{d} \backslash\{\boldsymbol{0}\}\right)$, we have $\mu_{1}=\mu / \mathrm{E}(N)$. This completes the proof.

\section{Acknowledgements}

The authors thank the anonymous referee for comments that led to a substantial improvement of the presentation of the material. The research of the first author was supported by the Swedish Research Council.

\section{References}

[1] BASRAK, B. (2000). The sample autocorrelation function of non-linear time series. Doctoral Thesis, Department of Mathematics, University of Groningen.

[2] Basrak, B., Davis, R. And Mikosch, T. (2002). A characterization of multivariate regular variation. Ann. Appl. Prob. 12, 908-920.

[3] Billingsley, P. (1968). Convergence of Probability Measures. John Wiley, New York.

[4] Billingsley, P. (1995). Probability and Measure, 3rd edn. John Wiley, New York.

[5] Braverman, M., Mikosch, T. and Samorodnitsky, G. (2002). Tail probabilities of subadditive functionals acting on Lévy processes. Ann. Appl. Prob. 12, 69-100.

[6] Cline, D. and Resnick, S. I. (1992). Multivariate subexponential distributions. Stoch. Process. Appl. 42, 49-72.

[7] Embrechts, P., Goldie, C. and Veraverbeke, N. (1979). Subexponentiality and infinite divisibility. Z. Wahrscheinlichkeitsth. 49, 335-347.

[8] De HaAn, L. and Lin, T. (2001). On convergence toward an extreme value distribution in C [0, 1]. Ann. Prob. 29, 467-483.

[9] Hult, H. And LindsKog, F. (2005). Extremal behavior for regularly varying stochastic processes. Stoch. Process. Appl. 115, 249-274.

[10] Hult, H. ANd LindsKog, F. (2006). Extremal behavior of stochastic integrals driven by regularly varying Lévy processes. To appear in Ann. Prob.

[11] Hult, H. And Lindskog, F. (2006). On Kesten's counterexample to the Cramér-Wold device for regular variation. Bernoulli 12, 133-142.

[12] Hult, H., Lindskog, F., Mikosch, T. and Samorodnitsky, G. (2005). Functional large deviations for multivariate regularly varying random walks. Ann. Appl. Prob. 15, 2651-2680.

[13] Kallenberg, O. (1983). Random Measures, 3rd edn. Akademie-Verlag, Berlin. 
[14] Lindskog, F. (2004). Multivariate extremes and regular variation for stochastic processes. Doctoral Thesis, Department of Mathematics, Swiss Federal Institute of Technology, Zürich. Available at http://e-collection. ethbib.ethz.ch/show?type=diss\&nr=15319.

[15] Pratt, J. (1960). On interchanging limits and integrals. Ann. Math. Statist. 31, 74-77.

[16] Protter, P. E. (2004). Stochastic Integration and Differential Equations, 2nd edn. Springer, Berlin.

[17] ReSNICK, S. (2004). On the foundations of multivariate heavy-tail analysis. In Stochastic Methods and Their Applications (J. Appl. Prob. Spec. Vol. 41A), Applied Probability Trust, Sheffield, pp. 191-212.

[18] ReSNick, S. I. (1986). Point processes, regular variation and weak convergence. Adv. Appl. Prob. 18, 66-138.

[19] Resnick, S. I. (1987). Extreme Values, Regular Variation, and Point Processes. Springer, New York.

[20] Rosiński, J. AND SAmorodnitsky, G. (1993). Distributions of subadditive functionals of sample paths of infinitely divisible processes. Ann. Prob. 21, 993-1014.

[21] Sato, K.-I. (1999). Lévy Processes and Infinitely Divisible Distributions (Camb. Stud. Adv. Math. 68). Cambridge University Press.

[22] Willekens, E. (1987). On the supremum of an infinitely divisible process. Stoch. Process. Appl. 26, $173-175$. 